\title{
Dispersal of an ancient retroposon in the TP53 promoter of Bovidae: phylogeny, novel mechanisms, and potential implications for cow milk persistency
}

Yaron Dekel ${ }^{1,4,5+}$, Yossy Machluf ${ }^{2 \dagger}$, Shifra Ben-Dor ${ }^{2}$, Oren Yifa ${ }^{2}$, Aviad Stoler ${ }^{4}$, Izhar Ben-Shlomo ${ }^{3^{*}}$ and Dani Bercovich ${ }^{4^{*}}$

\begin{abstract}
Background: In recent years, the perception of transposable genetic elements has changed from "junk DNA" to a focus of interest when appearing near or inside genes. Bov-A2 is a short interspersed nuclear element (SINE) that was first found in Bovidae and later in other ruminants. This retroposon is mostly used as a marker for phylogenetic analysis.

Results: We describe insertions of Bov-A2 in the promoter region of TP53, a key tumor suppressor gene that is indispensable for diverse developmental processes, in Antilopinae and Tragelaphini (belonging to the Bovinae subfamily). In Tragelaphini two Bov-A2 elements were inserted sequentially, whereas in 5 tribes of Antilopinae only one Bov-A2 element was inserted, in a different site and reverse orientation. The entrance site in both cases employed short palindromes that can form hairpin secondary structures. Interestingly, mutations that create or disrupt base pairing in the palindrome sequence dictated the presence or absence of Bov-A2, such as in the domestic cow and buffalo, which lack Bov-A2. Transcription factor binding site analysis revealed unique binding sites for STAT3 and NFKB within the Bov-A2 sequence, which together with TP53 itself are known to play a crucial role in mammary involution.

Conclusions: This report demonstrates how short palindromes serve as hot spots for Bov-A2 retroposon insertion into the mammalian genome. The strict correlation between point mutation in the palindromes and the presence/absence of Bov-A2 retroposon insertions, questions the use of singular insertion events as valid phylogenetic markers inside families. Bov-A2 insertion into the TP53 promoter in Antilopinae and Tragelaphini may not only provide a genetic network that regulates mammary involution, but can also answer the need for rapid mammary involution in Savanna antelopes after weaning, partially in response to predation stress. The absence of Bov-A2 in domestic bovids may constitute the molecular background for greater lactation persistency.
\end{abstract}

Keywords: TP53 Promoter, Bov-A2, Molecular Phylogeny, Milk Persistency, Palindrome

\section{Background}

Transposable genetic elements have been known for decades since the pioneering work of Barbara McClintock [1]. However, only in recent years, with the aid of novel massive parallel sequencing technologies and computational power, can these elements be more easily identified,

\footnotetext{
*Correspondence: izhar.benshlomo@gmail.com; dannyb@telhai.ac.il ${ }^{\dagger}$ Equal contributors

${ }^{3}$ Faculty of Medicine in the Galilee, Bar Ilan University \& Baruch Padeh Medical Center, Poria, \& Zefat Academic College, Safed, Israel

${ }^{4}$ Tel Hai College, Upper Galilee, Israel

Full list of author information is available at the end of the article
}

classified and studied $[2,3]$. The largest class of sequences in mammalian genomes is interspersed repetitive elements that can constitute, in some cases, more than $50 \%$ of the genome $[4,5]$. Retrotransposons and retrotransposon-like elements (RTE), such as retroposons, are the most common repeats. These elements replicate in a manner similar to retroviruses, via DNA transcription followed by reverse transcription of their RNA intermediate and finally DNA integration into the genome [6]. The abundance of ruminant-specific repeats, such as BovB, Bov-tA, 
Bov-A2 and ART2A, is negatively correlated with gene density and GC content [7].

Most retroposons and RTEs were considered nonfunctional and thus regarded as "junk DNA". At present, after gaining more information and understanding of control mechanisms, there are increasing indications that retroposons can serve different functions in the evolutionary process of exaptation [8]. Promoters, enhancers, silencers and chromatin modifiers are only part of the growing list of control mechanisms that have been reported to involve these elements [4]. Genomic repeats are particularly abundant in promoters and cis-regulatory elements, in particular transposable elements, which may serve as a rich source of material for the assembly of and tinkering with eukaryotic gene regulatory systems $[9,10]$. They may also be involved in post-transcriptional regulation of gene expression (for example, as small RNA and miRNA producers as well as controlling elements of RNA stability) and possibly even in translation regulation [11]. A growing body of evidence now indicates a close association of transposable elements with noncoding RNAs (ncRNA), which in turn may participate in a wide-range of regulatory functions, as well as be linked to disease [12]. By re-wiring regulatory networks, the mobilome (including RTEs) can facilitate the evolution of complex and novel physiological processes involving gene expression on a global scale. An intriguing example for this is the evolution of pregnancy [13].

Bov-A2 is a retroposon that was first discovered in bovid species and therefore was named after the family Bovidae [14]. It was later shown to be widely distributed among other ruminant genomes [15]. The element is composed of two Bov-A monomers which are joined together by a linker sequence, and ends with a $[A G C] n$ repeat $[16,17]$. Bov-A2 is usually categorized as a member of the short interspersed nuclear element (SINE) family $[11,14,18]$. Notably, Onami and colleagues proposed that due to the absence of promoter sequences for RNA polymerase III and the homology of its two units to the Bov-B long interspersed nuclear element (LINE), Bov-A2 does not belong to a SINE family but rather is a kind of retroposon that is transcribed by RNA polymerase II [16]. Events of SINE excision from its location in the genome are extremely rare, thus making it a suitable genetic marker for taxonomy [19]. Several studies on the distribution of Bov-A2 in the ruminant genome have been conducted. Bov-A2, along with other members of the Bov-A SINEs and Bov-B LINEs, constitutes an important marker in deciphering the evolution and phylogeny of ruminants $[11,19]$.

Bovidae, a family belonging to ungulate ruminants, consists of two subfamilies, Bovinae and Antilopinae. According to common classifications [20,21], these subfamilies are, respectively, of Eurasian and African origin
[22]. In total, the family comprises almost 140 extant species and more than 300 fossil species. However, the phylogenetic relationships and taxonomy of Bovidae are still controversial $[23,24]$. Bovidae include the economically most important species to mankind - the cow (Bos taurus), which was among the first livestock animals to be domesticated around 9000 B.C. [25,26], close to the time of the domestication of goats and sheep [27,28]. Recently, it was claimed that the former followed the later [29], though the order is still under genomic [30] and archaeological [31] research and debate. Due to its importance as the main milk and meat source in the world, the cow was the first livestock mammal whose genome was fully sequenced [32,33], and efforts are devoted to support annotation of the bovine genome [34].

Stemming from a search of differences in key regulatory genes, which might serve as the genetic background for domestication or adaptation to ecological niches, we analyzed the P1 promoter sequence of TP53, among others. Here, we report on the localization of Bov-A2 in the promoter region of the tumor suppressor gene TP53 in Bovidae. This SINE was inserted in reverse orientation into the TP53 P1 promoter of tribes belonging to Antilopinae, clearly defining this subfamily from Bovinae. The assumed mechanism of insertion was found to involve a palindromic conserved sequence in all Antilopinae tribes that we sequenced. Surprisingly, in Tragelaphini, a tribe belonging to the subfamily Bovinae, two Bov-A2 elements were inserted sequentially, in the original orientation and at a different site located 39 bp downstream to the Antilopinae insertion site. This probably occurred due to a second palindromic sequence that carries a mutation specific to spiral-horn antelopes and is absent from the genomes of the rest of the members of the family. In domestic cattle (cow and zebu, of European and Asian origin, respectively), Bov-A2 is absent from the TP53 P1 promoter region, most likely due to a point mutation in the first palindromic entrance site. Computational analysis of the ruminants' TP53 P1 promoter revealed putative RTE-specific transcription factor binding sites (TFBS) which cause physiological and evolutionary repercussions. We detail the findings and discuss the possible implications to the evolution of and phenotypic differences within the Bovidae.

\section{Results}

\section{Analysis of the TP53 P1 promoter of Bovidae}

The P1 promoter region of TP53 in the Bos taurus (domestic cow) genome was determined by transcript and EST analysis, and confirmed by alignment to the known TP53 P1 promoter in humans. The P1 promoter of TP53 is approximately $1.5 \mathrm{~kb}$ upstream to the TP53 transcription start site (TSS), which is composed of a 1088 nucleotide-long non-coding region and most of the 
WRAP53 first exon. The sequence of the P1 promoter was determined experimentally by PCR amplification, gel electrophoresis and subsequent sequencing. Sequence analysis revealed a product $272 \mathrm{bp}$ longer than the one found in the cow in all Antilopinae tribes analyzed in this study, including: ibex (Capra nubiana), goat (Capra aegagrus hircus), sheep (Ovis aries), wildebeest (Connochaetes taurinus), addax (Addax nasomaculatus) and gazelle (Gazelle gazelle) (Figure 1). The additional $272 \mathrm{bp}$ segment, which is located approximately $500 \mathrm{bp}$ upstream of the TP53 TSS, is not indicated in the published genome of sheep, as opposed to the goat genome (data not shown). Bioinformatics analysis identified the same additional 272 bp segment in the same location within the TP53 P1 promoter of Tibetan antelope (Pantholops hodgsonii), a species belonging to the Pantholopini tribe (Figure 1). In order to further investigate this evolutionary conservation among tribes belonging to the

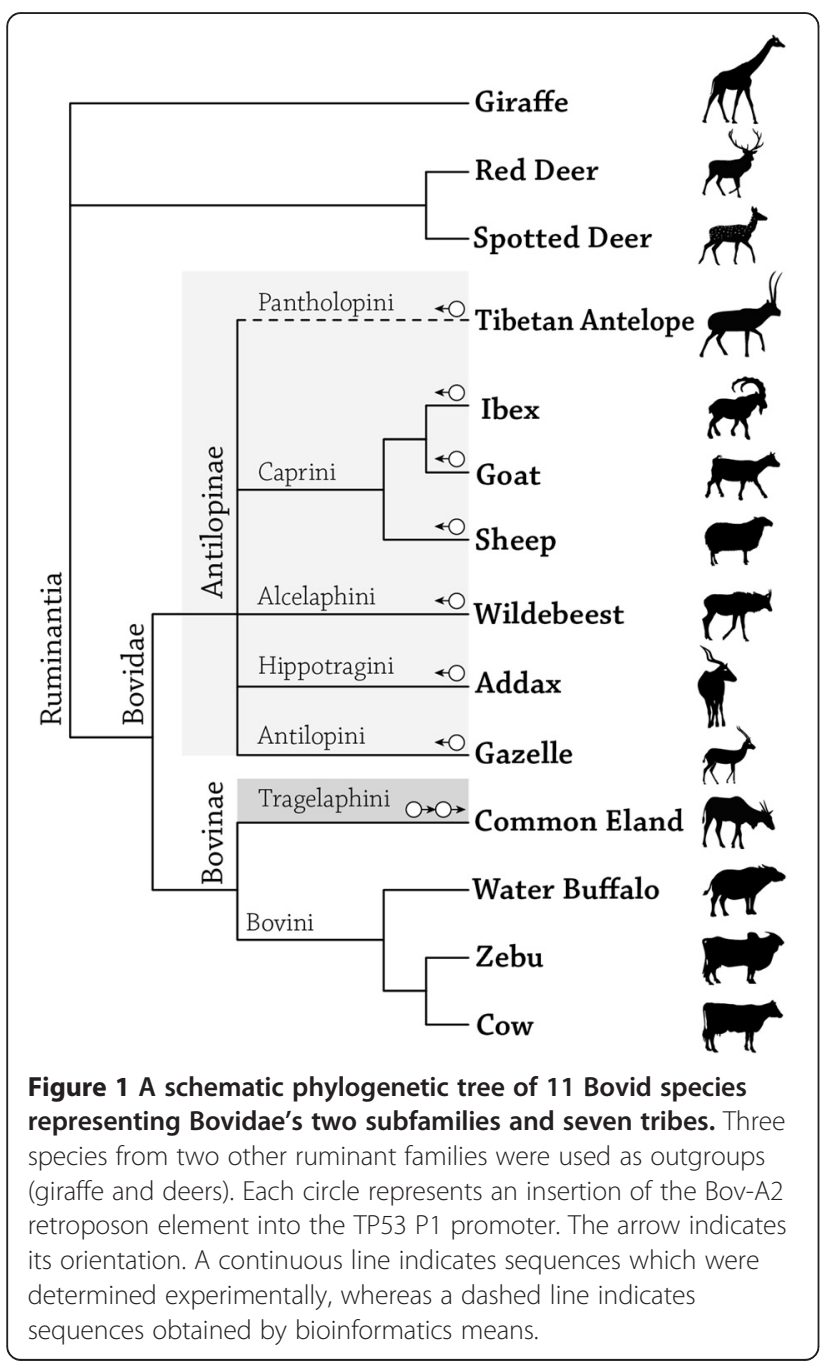

Antilopinae subfamily, we looked into the TP53 P1 promoter of three additional species of the Bovinae subfamily: one belonging to the Tragelaphini tribe (the common eland from spiral horned antelopes - Taurotragus oryx) and two belonging to the Bovini tribe (water buffalo Bubalus bubalis, and zebu - Bos indicus). In the zebu and buffalo, similarly to the cow, the extra $272 \mathrm{bp}$ long segment was not found, whereas in the common eland a segment of 522 bp was found in a location 39 bp downstream to the Antilopinae entrance site (Figure 1). It appears that the insertion into the TP53 P1 promoter, in terms of size and location, resulted from two independent and unrelated insertion events which occurred after the split between the two subfamilies (approximately 23 million years ago [22]): one ancient and common to all seven (analyzed) Antilopinae species, and one unique to the tribe Tragelaphini. Examination of the same promoter region in the outgroups in this study, two close families of bovidae, Cervidae (represented by red deer Cervus elaphus, and spotted deer - Axis axis) and Girraffidae (represented by giraffe - Giraffa camelopardalis), revealed no insertion, similar to the condition in Bovini (Figure 1).

\section{Characterization of the Bov-A2 element in the TP53 P1 promoter of Bovidae}

The additional 272 bp segment in Antilopinae is a SINE insertion that was previously designated as Bov-A2 [14]. The insertion site of Bov-A2, though different in Antilopinae and Tragelaphini, was within a LINE sequence in the TP53 P1 promoter, approximately $500 \mathrm{bp}$ and $460 \mathrm{bp}$ upstream to the TSS, respectively (Figure 2A). In all 8 Bovidae species, representing 6 tribes, that were experimentally and computationally explored here (Tibetan antelope was explored only bioinformatically), the conserved Bov-A2 elements were clearly defined. This retroposon element consists of a two Bov-A monomers connected by a $27 \mathrm{bp}$ linker, and ends with a repetitive terminator sequence $[\mathrm{AGC}] \mathrm{X}_{4}$. The two Bov-A monomers can be distinguished by polymorphisms at specific loci, termed diagnostic sites, as well as different lengths (the $1^{\text {st }}$ and $2^{\text {nd }}$ monomers are $116 \mathrm{bp}$ and $117 \mathrm{bp}$ long, respectively) [16]. Based on the Bov-A2 components and differences between the Bov-A monomers, one can determine that the Bov-A2 element entered once into the TP53 P1 promoter of the Antilopinae species (sheep, goat, ibex, addax, gazelle, wildebeest, and Tibetan antelope), in the reverse orientation, whereas two Bov-A2's (rather than Bov-A4) adjacent to each other entered the TP53 P1 promoter of Tragelaphini (common eland) in the forward orientation (Figure 2B).

In comparison with published data [16], some point mutations were found in the first and second Bov-A monomers, the linker and in one instance even in the 


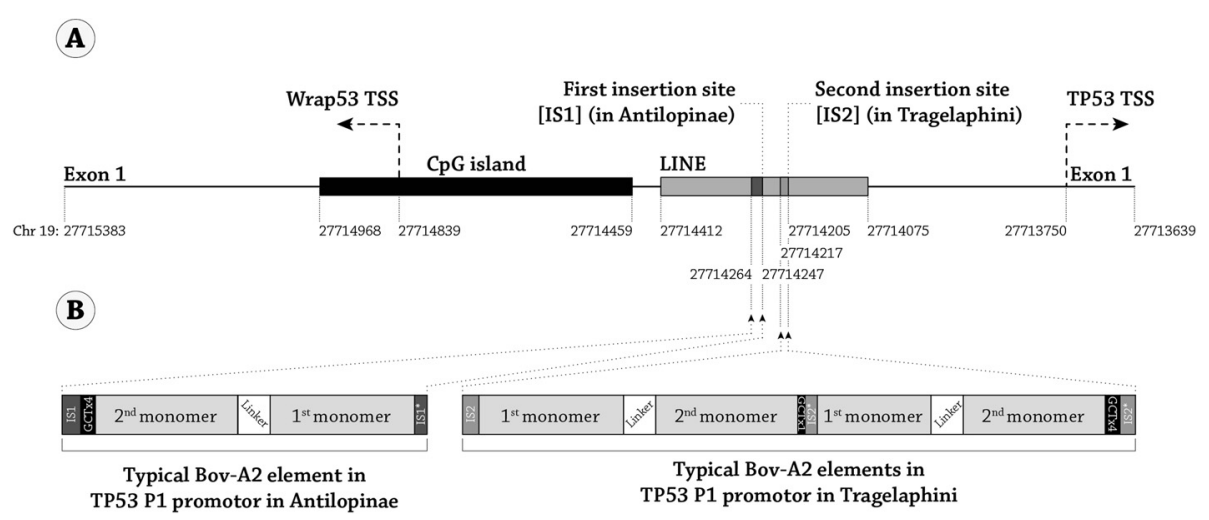

Figure 2 The insertion of Bov-A2 retroposon element into the TP53 P1 promoter. (A) A schematic representation of the genomic region of the TP53 P1 promoter in cow. The genomic cow chromosome 19 coordinates are indicated, based on the Baylor Btau_4.6.1/bosTau7 October 2011 assembly available in the UCSC genome browser (http://genome.ucsc.edu/cgi-bin/hgBlat?command=start). (B) The location, orientation and structure of the Bov-A2 retroposon element in the TP53 P1 promoter of Antilopinae (left) and Tragelaphini (right). Abbreviations: TSS- Transcription start site, IS1- Insertion site 1, IS2- insertion site 2 .

terminator sequence. The spread of the mutations in the different species and tribes, and their precise locations, are summarized in Figure 3. All of the mutations that are seen in the goat are also found in its wild relative the ibex. In gazelles, the same Bov-A2 mutations also appear, with one exception in the $2^{\text {nd }}$ monomer. In addition, there are 3 species-specific mutations, all in the $2^{\text {nd }}$ monomer. The wildebeest shares all its Bov-A2 mutations with the sheep, although they are from different tribes. As sheep, goat and ibex belong to the same tribe, it was unexpected that the sheep sequence would be more similar to the wildebeest sequence. Multiple sequence alignment of the full TP53 P1 promoter revealed that this finding is indeed valid, since in the whole promoter region the sheep sequence is more similar to the wildebeest (data not shown). The common eland, a member of the Bovinae subfamily, has unique mutations, mainly within the second downstream Bov-A2 retroposon element (closer to the TP53 TSS), that are not seen in its relatives from Antilopinae. This observation strengthens the notion of an independent Bov-A2 insertion event at the TP53 promoter of Bovidae (Figure 3).

\section{Palindromes act as recognition sequences for the Bov-A2 insertion}

In all of the studied species belonging to Antilopinae, there was one insertion event of the Bov-A2 element in the exact same location and yet in reverse orientation to the one described in the literature. The element resides between two similar sequences, TCAGAAAGTGAT (Figure 4A, left panel). The sequence at the 3 ' end is probably a result of non-equal homologous recombination during the insertion into the genome [35,36].
When looking broadly at the sequences of the insertion site, an 18 bp palindrome is detected (Figure 4A, right panel) and its sequence is fully conserved in 6 out of 7 species of Antilopinae. This palindrome will be designated here as PALI1. In the domestic cow (both European and Indian subspecies), a transversion mutation, $\mathrm{G}$ to $\mathrm{A}$, at the $14^{\text {th }}$ position of PALI1 results in a mismatch in base pairing (Figure 4) which in turn can impede the ability of this sequence to form three dimensional hairpin structures due to energetic constraints (Additional file 1: Figure S1, free energies [37] and DNA structures based on mfold Web Server [38]). Surprisingly, another member of the Bovini tribe, the water buffalo, which lacks a Bov-A2 sequence in the TP53 P1 promoter, has an intact PALI1 sequence and so its sequence is similar to its far relatives in Bovidae and dissimilar from its very close relative the cow. In the common eland, PALI1 is not mutated, and yet Bov-A2s were inserted 39 bp downstream from PALI1, at a different palindromic sequence, designated here as PALI2 (Figures 2A and $4 \mathrm{~B}$ ). In this species (Figure 4B, left panel), PALI2 has one extra nucleotide match due to a speciesspecific transversion point mutation, $\mathrm{A}$ to $\mathrm{C}$, and it lacks the last 4 nucleotides, both resulting in a different base-pairing and a more stable palindrome (Figure 4B, right panel and Additional file 1: Figure S1). The Bov-A2 elements were probably inserted here in two sequential events. In the first event, the insertion occurred following the PALI2 mutated sequence (or perhaps the 4 missing nucleotides are the result of this event). In the second event, the insertion occurred following the newly formed PALI2 sequence that appeared as a consequence of the first event. In both cases, the non-equal homologous 


\begin{tabular}{|c|c|}
\hline & Bov-A2 $1^{\text {st }}$ unit \\
\hline Bov-A2 consensus & \multirow{2}{*}{$\begin{array}{l}\text { - GGAGAAGGCAATGGCACCCCACTCCAGTacTCTTGCCTGGAaAATCCCAtGGACGGaGGAGCCTGGTaGGCTGCaGTCCATGGGGTCGCtaAGAGTCGGACACGACTGAGCGACTT } \\
\text { CAGAGAAGGCAATGGCACCCCACTTCAGTACTCTTGCCTGGAAAATCCCATGGATGGAGGAGCCTGGTAGGCTGCAGTCCATGGGGTCGCTAAGARTCGGACACGACTGAGCGACTT }\end{array}$} \\
\hline Tibetan Antelope & \\
\hline Ibex & CCGAGAAGGCAATGGCACCCCACTCCAGTACTCTTGCCTGGAAAATCCCATGGATGGAGGAGCCTGGTAGGCTGCAGTCCATGGGGTCGCTAAGAATCGGACACGACTGAGCGACTT \\
\hline Goat & CCGAGAAGGCAATGGCACCCCACTCCAGTACTCTTGCCTGGAAAATCCCATGGATGGAGGAGCCTGGTAGGCTGCAGTCCATGGGTTCGTAAGAATCGGACACGACTGAGCGACTT \\
\hline Sheep & CCGAGAAGGCAATGGCACCCCACTCCAGTACTCTTTCCTGGAAAATCCCATGGATGGAGGAGCCTGGTAGGCTGCAGTCCATGGGGTCGCTAAGAATCGĀACACGACTGAGCGACTT \\
\hline Wildebeest & CCGAGAAGGCAATGGCACCCCACTCCAGTACTCTTICCTGGAAAATCCCATGGATGGAGGAGCCTGGTAGGCTGCAGTCCATGGGGTCGCTAAGAATCGAACACGACTGAGCGACTT \\
\hline Addax & - GGAGAAGGCAATGGCACCCTIACTCCA\#TACTCTTGCCTGGAAAATCCCATGGATGGAGGAGCCTGGTAGGCTGCAGTCCATGGGGTCGCTAAGAATCGGACAGGACTGAGCGACTT \\
\hline Gazelle & - GGAGAAGGCAATGGCACCC凹ACTCCAGTACTCTTGCCTGGAAAATCCCATGGATGGAGGAGCCTGGTAGGCTGCAGTCCATGGGTCGCTAAGAATCGGACACGACTGAGCGACTT \\
\hline Common Eland-1 $1^{\text {st }}$ element & 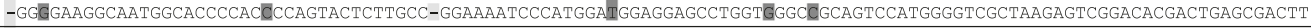 \\
\hline Common Eland-2 $2^{\text {nd }}$ element & 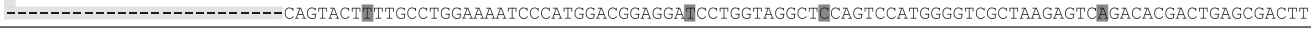 \\
\hline & Bov-A2 $2^{\text {nd }}$ unit \\
\hline Bov-A2 consensus & CACTTTCACTTTTCACTTTCATGCATTGGAGAAGGaAATGGCAaCCCACTCCAGTgtTCTTGCCTGGAgAATCCCAgGGACGGgGGAGCCTGGTgGGCTGCCGTCtATGGGGTCGCa \\
\hline Tibetan Antelope & СССTTTCACTTС̈TCACTTTCATGCATTGGAGAAGGAAATAెGCAACCCACTCCAGTGTTCTTGCCC̈GGAGAATCCCG̈GGGACGGGGAGCCTGGTGGGCTTCCGTCTATGGGGTCGCA \\
\hline Ibex & CDCTTTCACTTGTCACTTTCATGCATTGGAGAAGGAAATGGCAACCCATTCCAGTGTTCTTGCCCGGAGAATCCCGGGGACGGGGAGCCTGGTGGGCTMCCGTCTATGGGGTCGCA \\
\hline Goat & СССTTTCACTTGTCACTTTCATGCATTGGAGAAGGAAATGGCAACCCATTCCAGTGTTCTTGCCCGGAGAATCCCGGGGACGGGGAGCCTGGTGGGCTTCCGTCTATGGGGTCGCA \\
\hline Sheep & СССTTTCACTTTTCATTTTCATGCATTGGAGAAGGAAATGGCAACCCATTCCAGTGTTCTTGCCCGGAGAATCCCGGGGACGGGGAGCCTGGTGGGCTTCCGTCTATGGGGTCGCA \\
\hline Wildebeest & СССTTTCACTTTTCA TTTTCATGCATTGGAGAAGGAATGGCAACCCATTCCAGTGTTCTTGCCCGGAGAATCCCGGGGACGGGGGAGCCTGGTGGGCTMCCGTCTATGGGGTCGCA \\
\hline Addax & CACTTTGACTTTTCACTTTCATGCATTGGAGAAGGAAATGGCAACCCACTCCAGTGTTCTTGCCCGGAGAATCCCGGGGACGGGGGAGCCTGGTGGGCTMCCATCCATGGGGTCGCA \\
\hline Gazelle & СССTTTCACTTTTCACTTTCA_DGCATTG-AGAAGGAAATGGCAACCCACTCCAGTGTTCTTGCCCGGAGAATCCCGGGGACGGGGAGMCTGGTGGGCTMCCGTCTATGGGGTCGCA \\
\hline Common Eland- $1^{\text {st }}$ element & TCCTTTCACTTTTCACTTTMATGCATTGGAGAAGGAAATGGCAACCCACTCCAGTGTTCTTGCCTGGAGAATCCCAGGGACGGGGAGCCTGGTGGGCTGCCGTCTATGGGGTCGCA \\
\hline Common Eland $-2^{\text {nd }}$ element & CAMTTTCACTTTTCACTTTCATGCATTGGAGAAGGAAATGGCAACCCACTCCAGTGTTCTTGCCTGGAGAATCCCAGGGACAGCAGAGCTGGATAGGCTGCCGTCTATGGGGTCGCA \\
\hline \multicolumn{2}{|r|}{ Bov-A2 termination } \\
\hline \multicolumn{2}{|r|}{$\neg$ repeats $\neg$} \\
\hline Bov-A2 consensus & CAGAGTCGGACACGACTGAaGCGACTTAGCAGCAGC AGC \\
\hline Tibetan Antelope & CAGAGTTGGACACGACCGAAGCGATTTAGCAGCAGC AGC \\
\hline Ibex & CAGAGTCGGACACGACTGAAGCGATTTAGCAGCAGC AGC \\
\hline Goat & CAGAGTCGGACACGACTGAAGCGATTTAGCAGCAGC AGC \\
\hline Sheep & CAGAGTCGGACACGACTGAAGCGATTTAGCAGCAGC AGC \\
\hline Wildebeest & CAGAGTCGGACACGACTGAAGCGAMTTAGCAGCAGC AGC \\
\hline Addax & CAGAGTCGGACACGACTG----------AGCAGCAGC»AGC \\
\hline Gazelle & CAGA--------------AGCGACTTAGCAGCAGC AGC \\
\hline Common Eland- $1^{\text {st }}$ element & CAGAGTCGGACACGACTGAAGTGACTTAGC------- --- \\
\hline Common Eland-2 $2^{\text {nd }}$ element & CAGAGTCGGACACGACTGAAGCGACTTAGCAGCAGC AGC \\
\hline
\end{tabular}

Figure 3 Multiple alignment of Bov-A2 sequences found in TP53 P1 promoter. The Bov-A2 consensus sequence is based on literature survey (Onami et al. [16]), and its components' boundaries are marked. All sequences were determined experimentally, except the Tibetan antelope which was discovered by computational means. Mutations are highlighted: deletion (-) in light gray, nucleotide change in dark gray, and insertion in intermediate gray (a unique insertion in addax in marked by asterisk, and the sequence is depicted at the end of the line). Reverse complement sequences are shown for these species in which the Bov-A2 was inserted in reverse orientation (antelope, ibex, goat, sheep, wildebeest, gazelle).

recombination resulted in the formation of an additional flanking truncated PALI2 sequence. In the common eland, the existence of a truncated PALI2 sequence (the second site) further supports the notion of two adjacent Bov-A2 elements rather than a single Bov-A4 element. It is interesting to note that in the case of the single Antilopinae insertion event, the truncated repeat of PALI1 is truncated only in its $5^{\prime}$ side. In the case of the common eland, PALI2 is truncated at both the $5^{\prime}$ and the $3^{\prime}$ sides.

There is evidence for a second SINE insertion event in the addax. A unique polyA (more than 10 nucleotides long) is followed by a partial PALI1 sequence, all located between the third and last AGC triplet in the terminator of the first inserted Bov-A2 (Figure 3).

\section{Transcription factor binding site analysis of the TP53 P1 promoter in Bovidae}

Transcription factor binding site (TFBS) analysis was performed using the Genomatix Genome Analyzer
(GGA) MatInspector program [39]. To this end, the TP53 P1 promoter was analyzed, including the full CpG island (starting within the WRAP53 first exon) until 50 nucleotides downstream of the TP53 TSS (Figure 2), covering at least $1270 \mathrm{bp}$ in the case of SINE-less animals. Raw data for this analysis can be found in Additional file 2: Figure S2. The analysis focused on TFBS sequences that were newly brought into the promoter region by BovA2. The Antilopinae Bov-A2 element contains some unique TFBSs, such as DINR (twice), ZNF219, NFkB, NANOG, BCL6 and STAT1, that do not appear outside the Bov-A2 element in the remaining TP53 P1 promoter of Antilopinae or of other Bovini and Cervidae members (Figure 5). The first three TFBSs (DINR, ZNF219 and NFkB) also appear in the Bov-A2 element of Tragelaphini, whereas the last two TFBSs (BCL6 and STAT1) are unique to the sheep and wildebeest. STAT3 appears in most Antilopinae members only in the Bov-A2 element. Only in the addax and gazelle can an additional and conserved 


\begin{tabular}{|c|c|c|}
\hline \multirow[b]{2}{*}{ Bov-A2 } & \multicolumn{2}{|c|}{ First insertaion site } \\
\hline & Species & Sequence \\
\hline \multirow[t]{2}{*}{ No entrance } & Red Deer & ATCACTTCAGAAAGTGAT \\
\hline & Spotted Deer & ATCACTTCAGAAAGTGAT \\
\hline \multirow{15}{*}{$\begin{array}{l}\text { One site, } \\
\text { Reverse orientation } \\
\text { The entrance site }\end{array}$} & \multirow[t]{2}{*}{ Tibetan Antelope } & ATCACTTCAGAAAGĀGAT \\
\hline & & TCAGAAAGTGAT \\
\hline & \multirow[t]{2}{*}{ Goat } & ATCACTTCAGAAAGTGAT \\
\hline & & TCAGAAAGTGAT \\
\hline & \multirow[t]{2}{*}{ Ibex } & ATCACTTCAGAAAGTGAT \\
\hline & & TCAGAAAGTGAT \\
\hline & \multirow[t]{2}{*}{ Sheep } & ATCACTTCAGAAAGTGAT \\
\hline & & TCAGAAAGTGAT \\
\hline & \multirow[t]{2}{*}{ Wildebeest } & ATCACTTCAGAAAGTGAT \\
\hline & & TCAGAAAGTGAT \\
\hline & \multirow[t]{3}{*}{ Addax } & ATCACTTCAGAAAGTGAT \\
\hline & & GAAAGAAAGTGAT \\
\hline & & TCAGAAAGTGAT \\
\hline & \multirow[t]{2}{*}{ Gazelle } & ATCACTTCAGAAAGTGAT \\
\hline & & TCAG----TGAT \\
\hline$\underline{\underline{T} \text { wo }}$ sites, $1^{\text {st }}$ site & Common Eland & ATCACTTCAGAAAGTGAT \\
\hline \multirow[t]{3}{*}{ No entrance } & Water Buffalo & ATCACTTCAGAAAGTGAT \\
\hline & Zebu & ATCACT TCAGAAAAT TAT \\
\hline & Cow & ATCACTTCAGAAAATGAT \\
\hline
\end{tabular}

$$
\begin{gathered}
\text { A proposed secondary } \\
\text { structure of the first } \\
\text { insertaion site } \\
\text { marked is a critical } \\
\text { mutation in Cow and Zebu) }
\end{gathered}
$$

\begin{tabular}{|c|c|c|}
\hline \multicolumn{3}{|c|}{ Second insertaion site } \\
\hline Bov-A2 & Species & Second entrance site \\
\hline \multirow[t]{2}{*}{ No entrance } & Red Deer & \begin{tabular}{|l} 
GGTGTAATACAGCGCATATIT \\
\end{tabular} \\
\hline & Spotted Deer & GGTGTAATACAGCGCATATE \\
\hline \multirow{7}{*}{$\begin{array}{l}\text { One site, } \\
\text { Reverse orientation } \\
\text { Not an entrance site }\end{array}$} & Tibetan Antelope & \begin{tabular}{|l|} 
GGTGAAATATAGCGCATATG \\
\end{tabular} \\
\hline & Goat & \begin{tabular}{|l|} 
GGTGTAATATAGCGCATATC \\
\end{tabular} \\
\hline & Ibex & GGTGTAATATAGCGCATATG \\
\hline & \begin{tabular}{|l|} 
Sheep \\
\end{tabular} & \begin{tabular}{|l} 
GGTGTAATATAGCGCATATI \\
\end{tabular} \\
\hline & Wildebeest & GGTGTAATATAGCGCATATT \\
\hline & Addax & GGTGTAATATAGGGGATATC \\
\hline & Gazelle & GGTGTAATATAGCGCATATIT \\
\hline Two sites, $2^{\text {nd }}$ site & Common Eland & \begin{tabular}{|l|} 
GGTGTAATATAGCGCC---- \\
-GGTAATATAGMGG----- \\
----- - AATACAGCGCATATG
\end{tabular} \\
\hline \multirow[t]{3}{*}{ No entrance } & Water Buffalo & GGTGTAATATAGCGCATATE \\
\hline & Zebu & GGTGTAATATAGCGCATATG \\
\hline & Cow & \begin{tabular}{|l|} 
GGTGTAATATAGCGCATATC \\
\end{tabular} \\
\hline
\end{tabular}

(B)
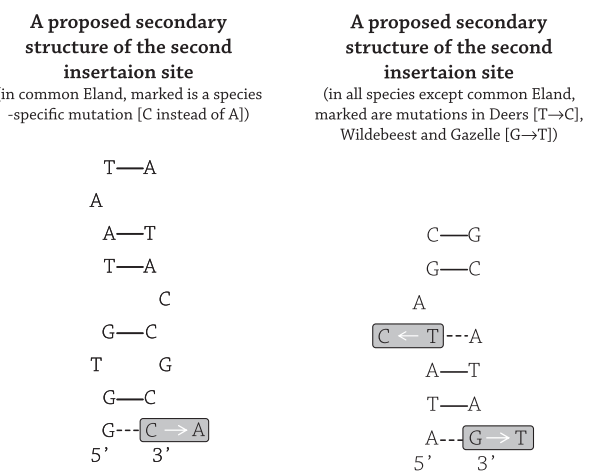

Figure 4 Multiple alignment (left) and proposed secondary structure (right) of the first (A) and second (B) Bov-A2 insertion sites in TP53 P1 promoter. Mutations are highlighted: deletion (-) in light gray, and nucleotide change in dark gray. Nucleotides in positions that pair in the secondary structure are marked in bold.

STAT3 TFBS be found near the TP53 TSS, as appears also in the Cervidae and other Bovids (Figure 5). Smad3 appears only in the gazelle Bov-A2 and not in any other species in the promoter region screened here. In terms of TFBS which are enriched in the Bov-A2 element, TAL1 appears twice in the Bov-A2 single insertion and 3 times in the Tragelaphini double insertion, and only once in the distal CpG island of all the animals sequenced here.

\section{Discussion}

A significant portion of mammalian genomes consists of repetitive DNA sequence fragments, such as retroposons in the forms of either short or long interspersed elements (SINEs and LINEs, respectively). Because of the clear presence of an element and the low likelihood of exact parallel insertions or deletions, the retroposon presence or absence is considered to be exceptionally effective for detecting ancestral lineages [40]. In the case of bovids, the retroposon Bov-A2, a SINE which is widely distributed in ruminants' genomes, is detected and used as a genetic marker for evolutionary classification. It was previously shown that very few insertion events, sometimes even a single event, can be used for classification in this important family in which classification is still under debate [19]. Additionally, in different organisms different classes of retro-elements show diverse integration site preferences into gene-rich regions $[41,42]$. The insertion event of a Bov-A2 element in the TP53 P1 promoter of Bovidae described here questions this general assumption. As we report, at the same exact location in the same family, a point mutation dictates the absence of Bov-A2 in the domestic cow and thus, when compared with species from other families, can lead to the assumption that the domestic cow is closer to Cervidae species which lack Bov-A2 at this location as well. In the case of Tragelaphini, one of Bovinae's tribes, two insertion events were evident yet at a different location from the rest of the family, 39 bp downstream to the common Antilopinae insertion site. As will be described shortly, the reason for the different 


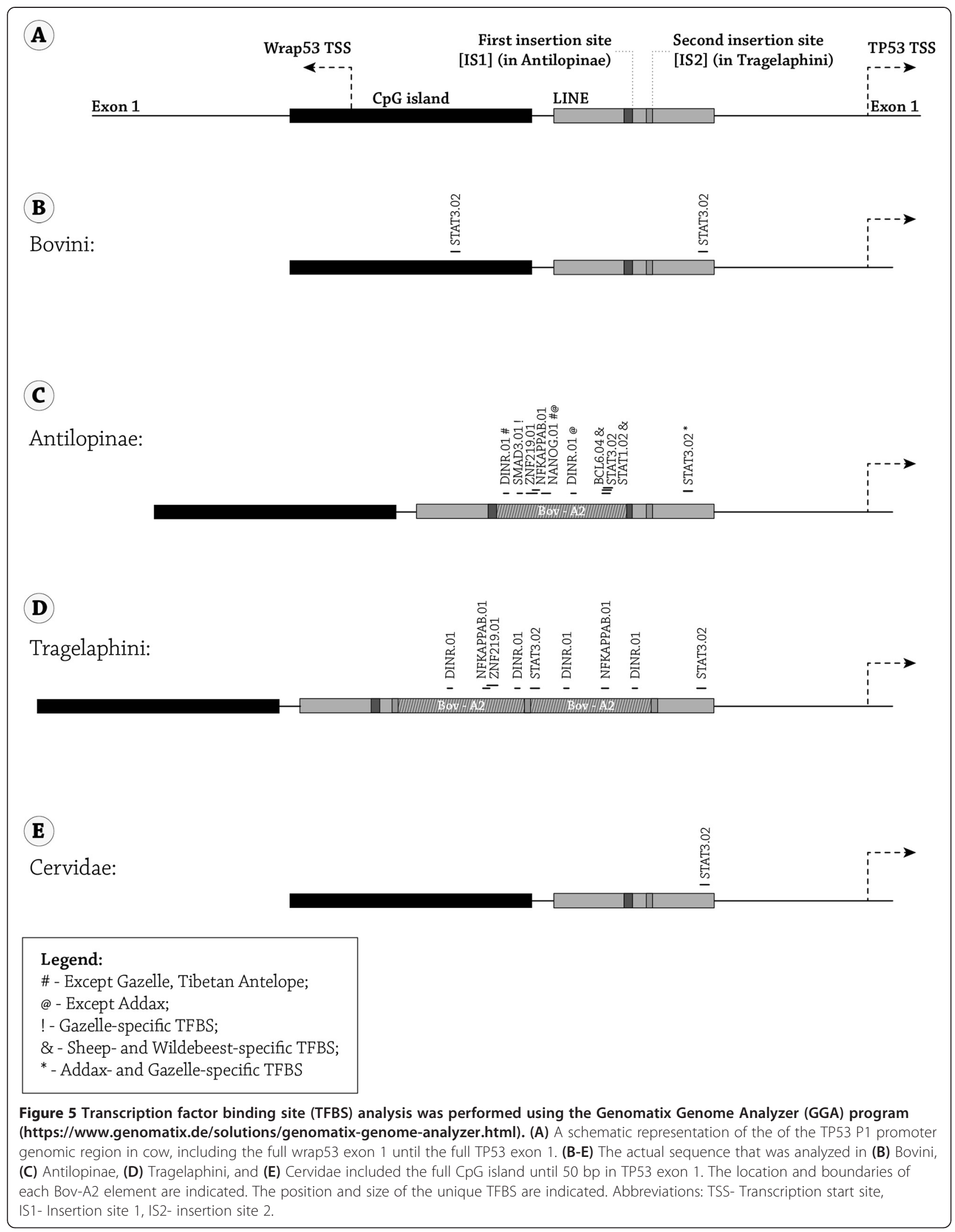


location and double insertion is sequence related and we argue that it is dictated by a single point mutation. Point mutations, the simplest genetic alteration, can thus lead to different retroposon dispersal in very close species and can mislead evolutionary scientists that use these single events as genetic markers for phylogenetic analysis.

The insertion event that took place in the Antilopinae subfamily is found similarly in all of the explored Antilopinae tribes, indicating that this event is very ancient and dates back at least 23MYA, to Antilopinae pre-speciation time [22]. This insertion event in a very important genomic "strategic" point might have had a significant functional value for the first Antilopinae representatives that migrated from Euro-Asia to Africa [23]. The double insertion events in Tragelaphini obviously took place after Bov-A2 inserted to Antilopinae' TP53P1 promoter, around 14MYA, as Bovini members (the cow/ buffalo tribe) lack Bov-A2 in TP53 P1 promoter [43]. In terms of evolutionary significance, it is interesting to note that a Tragelaphini member (the common eland), which according to the fossil record appeared in Africa between 5.5 MYA to 10.5 MYA [43], has two sequential Bov-A2 elements in the TP53 P1 promoter. In that sense, the common eland is more similar to its African relatives and neighbors in the savanna, the Antilopinae subfamily that have one Bov-A2 element, rather than to its own Euro-Asian subfamily relatives that lack it [23]. This might be a case of convergent evolution in which species from different subfamilies share a common trait due to similarity in their ecological niche.

The Bov-A2 element that was found here is mostly very similar to the previously described sequence in the literature [16]. The major differences are highlighted in Figure 3. When looking into these mutational differences and comparing them to the Bov-A2 consensus, one can clearly identify 4 mutations that are unique to Antilopinae (including the Tibetan antelope), 2 mutations that can separate Caprini (including the Tibetan antelope) and the wildebeest from the Antilopinae sequence, and one mutation unique to Caprini and the wildebeest. The sheep and wildebeest have 3 unique mutations, and the addax and gazelle share one unique mutation. Along with the above similarities, each inspected species has its own unique mutations, apart from the ibex and goat which have identical sequences. The Tibetan antelope, an endangered species from the Tibetan Plateau, is gaining scientific and medical attention due to its ability to live in hypoxic conditions and with high levels of ultraviolet radiation [44]. While there is some debate over the phylogenetic relationship of Tibetan antelopes, our data support the notion that the Tibetan antelope is a part of Antilopinae and more closely related to Caprini [45].
The insertion of Bov-A2 in unrelated insertion events in Antilopinae and Tragelaphini was made possible due to short palindromic sequences that can form small stem and loop structures, designated as PALI1 and PALI2. While PALI1, the entrance site for the Antilopinae BovA2 element in the TP53 P1 promoter, is highly conserved throughout the subfamily and beyond (Tragelaphini as well as in the outgroups, Giraffidae and Cervidae), in the domestic cow (both European and Indian) a G to A transversion mutation occurred and probably interfered with the ability of this palindromic sequence to form a stem and loop structure (Figure 3 and Additional file 1: Figure S1). This point mutation might be the cause of the absence of Bov-A2 in the extinct founder of the Bovini. As mentioned before, the insertion event in Antilopinae probably took place very early during Bovidae divergence into two subfamilies. The fact that this mutation occurred only in Bovini might have a significant role in this tribe's evolution. In the case of the water buffalo, which also belongs to Bovini, the existence of the full PALI1 is probably due to a later reversal mutation back to the original sequence as it lacks the Bov-A2 element, like its tribal fellows the cows. The entrance site in Tragelaphini is a $16 \mathrm{bp}$-long palindromic sequence, PALI2. This sequence is unique in the common eland and lengthens a shorter and thus less energetically favorable $14 \mathrm{bp}$ palindrome that exists in all bovid species. It is hard to speculate whether Bov-A2 entered after PALI2, 39 bp downstream to PALI1, because PALI2 is more "transposon favorable" or that as in the case of the water buffalo its original PALI1 sequence was mutated (like in Bovini) and only later reversed to the more functional one. It was reported earlier that palindromic AT rich sequences can be responsible for genome rearrangements [46] and transposon reintegration [47]. Although the former reported sequences are hundreds of nucleotides in length, and the latter is related to the Tol2 transposon, these findings support our proposed model, with PALI1 and PALI2 being the first reported cases of short palindromic sequences responsible for SINE insertion.

Another interesting finding, related to the insertion site of these Bov-A2 elements into the TP53 P1 promoter, is the mechanism of insertion. It is well known that SINE elements need LINE elements to re-transpose and they do so through an unequal crossing over mechanism [48]. In the case of both Bov-A2 insertions reported here (in Bovinae and Tragelaphini), a partial palindromic sequence was replicated and is evident adjacent to the 3' end of the Bov-A2 element. This partially replicated palindromic sequence was sufficient in the case of the common eland to enable a second Bov-A2 insertion, resulting in two sequential Bov-A2 elements, rather than Bov-A4 [16]. In the case of the addax, we 
speculate that the partial PALI1 followed by a long poly (A) tail is the result of a second unsuccessful SINE insertion event (probably not Bov-A2 due to the poly(A) sequence) as poly(A)'s are part of known SINE insertion mechanisms into the genome [49]. Putting together the single insertion event in Antilopinae, the dual insertion event in Tragelaphini, and the second insertion attempt in the addax (a member of Antilopinae) all in the TP53 P1 promoter, strengthens the hypothesis that this promoter and its short palindromic sequences are hotspots for insertion of genetic elements, which probably provided an evolutionary advantage due to functional significance.

TFBS analysis revealed unique TFBSs that exist only in the inserted Bov-A2 elements and thus might represent a classic case of modified regulation of gene expression which may lead to modified function. The loss of the conserved STAT3 binding site in the LINE sequence of Antilopinae (apart from the wildebeest and sheep which retained this TFBS) and the gain of this same binding site in the Bov-A2 element in proximity to an NFkB binding site, might be a result of a subfunctionalizationlike process. As was mentioned above, in the common eland's TP53 promoter, two Bov-A2 insertion events were obtained, in very close proximity to the Antilopinae's Bov-A2 insertion site. This might be a case of convergent evolution in which African members of Bovidae, along with members that live in mountainous rocky regions northern to Africa (Caprinae), have a similar genomic alteration. It was shown experimentally that deletion of STAT3 or its conditional knockout in mice suppresses epithelial apoptosis and dramatically delays mammary gland involution upon forced weaning in mice [50-52]. Moreover, STAT3 along with NFkB signaling pathways are also responsible for inflammatory signaling and acute phase response that are part of later phases of the normal involution process [53], and were shown to be suppressed by Sim 2 which promotes delayed involution [54]. TP53 itself is induced rapidly following weaning of neonates [55]. BALB/c-TP53 null mice displayed delayed involution of the mammary epithelium [56]. TP53 is a physiological regulator of mammary involution that acts to rapidly initiate apoptosis in the secretory epithelium [57]. The combined loss of TP53 and STAT3 in double knockout mice, led to a severe perturbation in the mammary gland involution, with hyper delayed loss of epithelium and reappearance of adipocytes. In the absence of STAT3 alone, the apoptotic program can default to a p53 pathway [58]. Activation of $\mathrm{NFKB}$ in mammary epithelium promotes milk loss during mammary development [59]. In light of these and our TFBS analysis findings, one may propose that not only STAT3, NFkB and TP53 are involved in the regulation of involution, but also the interplay between these pathways, as the former two TF (presumably via an OR gate) regulate TP53 transcription during the weaning process.

Involution is a complex physiological mechanism, responsible for milk arrest and mammary remodeling upon weaning. Involution was vastly explored molecularly due to its importance to female breast health and due to its economic importance to the dairy market [53]. Although much of our present knowledge of involution, mainly its cellular and underlying molecular mechanism, is based on work with rodents, similarities to dairy animals and studies in bovine explants further support our model. It was shown that in goats and ewes the process of involution has both a similar timeline as well as parallel stages to what is seen in rodents $[60,61]$. The situation in cows is different as the incidence of apoptosis during tissue remodeling between lactations appears markedly lower [61]. Mammary involution proceeds with little loss of epithelial cells, and no disengagement of epithelial cells from the basement membrane [62]. Mammary explants from pregnant dairy cows have the capacity to maintain a population of surviving mammary epithelial cells that remain hormone-responsive, are capable of milk protein gene expression, and maintain alveolar architecture without exposure to exogenous macromolecules during culture [63]. This intrinsic capacity was attributed to expression of genes which lead to avoidance of cell death pathways [63]. It was proposed that identification of genes that may contribute to cell survival in bovine mammary explants may constitute a significant financial advantage for the dairy industry, due to improved persistence of lactation [64]. These observations, in light of our results, raise the possibility that the absence of Bov-A2 from the TP53 P1 promoter of Bovini domestic species might be the cause for delayed mammary involution.

It is intriguing to speculate that the African Savanna and steep high cliff niches demand its herbivores to wean rapidly and return to normal morphology as soon as possible. The strong predation stress in the Savanna, and the harsh topography that members of Caprini inhabit as compared to the Euro/Asia Bovid habitat, can be strong stressors that favor a shorter involution period. Altogether this might explain the ability of our domestic cows and buffalos to bear greater persistency of lactation.

\section{Conclusions}

We report the independent insertions of Bov-A2 retroposons in the promoter region of TP53, the guardian of the genome, in Antilopinae and Tragelaphini. Whereas these Bov-A2 insertions differ in the genomic location of insertion site, orientation and number of inserted elements, they share a common feature: the entrance site in both cases employed short palindromes that can form 
hairpin secondary structures. To the best of our knowledge, this is the first report in which short palindromes serve as hot spots for retroposon insertion into the mammalian genome. TFBS analysis of TP3 promoter in Bovidae revealed that the Bov-A2 elements harbor unique binding sites for transcription factors that might regulate TP53 expression and mammary involution. This in turn may answer the need for rapid mammary involution in Bovidae species of the Savanna/mountainous regions. The absence of Bov-A2 in the TP53 promoter of domestic Bovids might have been the early background for future milk persistency.

\section{Methods}

\section{Biological samples}

Biological samples were taken from the following ruminants: Ibex (Capra nubiana, $\mathrm{n}=5$ ), Goat (Capra aegagrus hircus, $\mathrm{n}=5$ ), Sheep (Ovis aries, $\mathrm{n}=2$ ), Wildebeest (Connochaetes taurinus, $\mathrm{n}=1$ ), Addax (Addax nasomaculatus, $\mathrm{n}=1$ ), Gazelle (Gazelle gazelle, $\mathrm{n}=2$ ), Common Eland (Taurotragus oryx, $\mathrm{n}=1$ ), Water Buffalo (Bubalus bubalis, $\mathrm{n}=8$ ), Zebu (Bos indicus, $\mathrm{n}=4$ ), Cow (Bos taurus, $\mathrm{n}=6$ ), Red Deer (Cervus elaphus, $\mathrm{n}=3$ ), Spotted Deer (Axis axis, $\mathrm{n}=3$ ) and Giraffe (Giraffa camelopardalis, $\mathrm{n}=2$ ). Blood or hair roots were taken from domestic animals, whereas tissue samples were collected from deceased wild animals from the Tel Aviv Ramat Gan Zoological Center. The ibex tissue samples were a generous gift from the collection of Dr. Gila Kahila Bar-Gal from the Hebrew University of Jerusalem.

\section{Ethics statement}

Biological samples were collected by qualified and authorized personnel from accredited institutions, such as the chief veterinarian of the Tel Aviv Ramat Gan Zoological Center, and competent veterinarian authorities at dairy farms and petting zoos.

The biological samples used in this non-experimental research were collected, packed, stored and treated following required standards and appropriate permissions from all relevant parties. According to the Israeli law, the samples collected from animals comply with institutional and national guidelines. Blood samples were taken by authorized veterinarians, as part of routine health procedures, and small portions were shared. Sharing tissues from abattoirs and dead animals do not require the approval of the Institutional Animal Care and Use Committee.

\section{DNA extraction, amplification and sequencing}

DNA was extracted from tissues, blood or hair using the QIAGEN DNA Isolation kit (QIAGEN, Germany).

PCR reactions were carried out in a total volume of 50 microliters with $10 \mathrm{mM}$ Tris $-\mathrm{HCl}, \mathrm{pH} 8.3,1.5 \mathrm{mM}$ $\mathrm{MgCl}_{2}, 50 \mathrm{mM} \mathrm{KCl}, 0.2 \mathrm{mM}$ dNTPs, and $30 \mathrm{nM}$ primers.
The PCR conditions were $94^{\circ} \mathrm{C}$ for $5 \mathrm{~min}$, followed by 35 cycles with annealing temperatures of $58^{\circ} \mathrm{C}$ (depending on the species) for $0.5 \mathrm{~min}$ and $72^{\circ} \mathrm{C}$ for $0.5 \mathrm{~min}$; the reactions were ended with a final extension step at $72^{\circ} \mathrm{C}$ for $7 \mathrm{~min}$.

Three sequential primers pairs were used to amplify 1500 bp upstream of the TP53 P1 promoter, each pair designed to amplify 500 bp with overlapping sequences. Primers were designed according to BOVIN ENSBTAT00000001420:

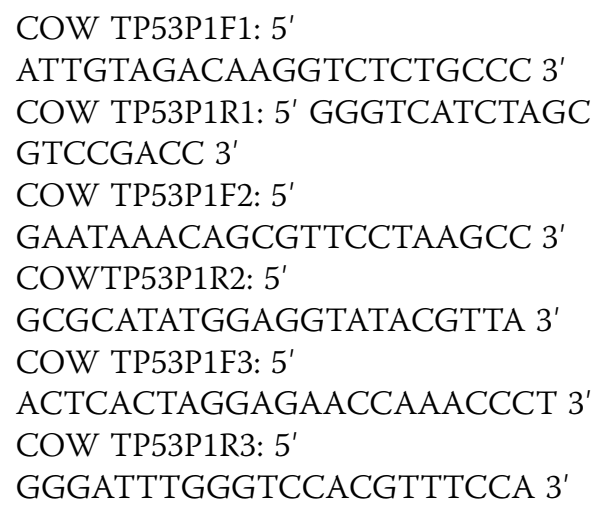

PCR products were Sanger sequenced and analyzed on ABI Sequencer (3100) using GeneScan and Genotyper software (PE Applied Biosystems).

The nucleotide sequences were deposited in GenBank (accession numbers: KM233178-KM233190). The Tibetan antelope (Pantholops hodgsonii) sequence (from the database) is AGTT01151016.

\section{Free energy calculation}

Free energies [37] and DNA structures were calculated based on the Mfold Web Server [38].

\section{Transcription factor binding site analysis}

Transcription factor binding site (TFBS) analysis was performed using the Genomatix Genome Analyzer (GGA) MatInspector program [39].

\section{Additional files}

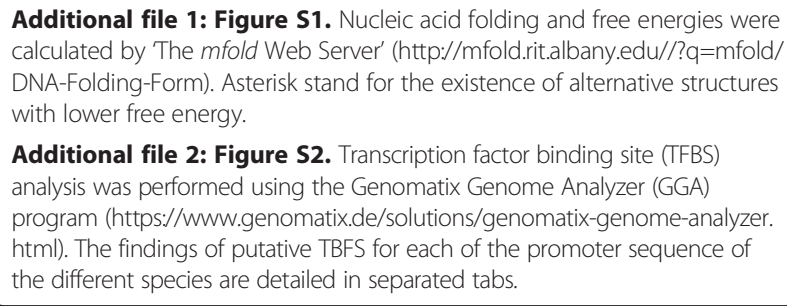

Additional file 1: Figure S1. Nucleic acid folding and free energies were calculated by 'The $m$ fold Web Server' (http://mfold.rit.albany.edu//?q=mfold/ DNA-Folding-Form). Asterisk stand for the existence of alternative structures with lower free energy.

Additional file 2: Figure S2. Transcription factor binding site (TFBS) analysis was performed using the Genomatix Genome Analyzer (GGA) program (https://www.genomatix.de/solutions/genomatix-genome-analyzer. html). The findings of putative TBFS for each of the promoter sequence of the different species are detailed in separated tabs.

\section{Abbreviations}

BCL: B-Cell CLL/Lymphoma; GGA: Genomatix Genome Analyzer; LINE: Long interspersed nuclear element; NFkB: Nuclear factor kappa B; SINE: Short 
interspersed nuclear element; STAT: Signal transducers and activators of transcription; TFBS: Transcription factor binding site; TSS: Transcription start site; ZNF: Zinc finger.

\section{Competing interests}

The authors declare that they have no competing interests.

\section{Authors' contributions}

YD carried out samples collection, sequencing, sequence alignment and drafted the manuscript. YM participated in the sequence alignment, carried out all bioinformatics analyses and drafted the manuscript. SBD assisted with computational analysis genomic databases and bioinformatics analyses. OY performed DNA purifications and PCRs. AS performed DNA purifications and PCRs. YBS helped to draft the manuscript. DB assisted with the design and coordination of this work. All authors read and approved the final manuscript.

\section{Acknowledgments}

The authors would like to thank Dr. Igal Horowitz and his team from the Tel Aviv Ramat Gan Zoological Center for their help in supplying biological samples from wild ruminants. We wish to thank Dr. Gila Kahila Bar-Gal from the Hebrew University of Jerusalem for allowing us to use biological samples of Ibexes from her collection. The graphics in this paper were accomplished with the kind help of Ziv Ariely from the Department of Science Teaching, Weizmann Institute of Science. The authors thank Rachel Teitz for linguistic editing of the manuscript. This work was supported by G.G.A - Galil Genetic Analysis Ltd, Kazerin, Israel (http://www.gga.org.il/homepage).

\section{Author details}

${ }^{1}$ MIGAL - Galilee Research Institute, Kiryat Shmona, Israel. ${ }^{2}$ Weizmann Institute of Science, Rehovot, Israel. ${ }^{3}$ Faculty of Medicine in the Galilee, Bar Ilan University \& Baruch Padeh Medical Center, Poria, \& Zefat Academic College, Safed, Israel. ${ }^{4}$ Tel Hai College, Upper Galilee, Israel. ${ }^{5}$ Golan Research Institute, Katzrin, Israel.

\section{Received: 28 September 2014 Accepted: 12 January 2015} Published online: 05 February 2015

\section{References}

1. Fedoroff NV. McClintock's challenge in the 21st century. Proc Natl Acad Sci U S A. 2012;109:20200-3.

2. Nakagawa S, Bai H, Sakurai T, Nakaya Y, Konno T, Miyazawa T, et al. Dynamic evolution of endogenous retrovirus-derived genes expressed in bovine conceptuses during the period of placentation. Genome Biol Evol. 2013;5:296-306

3. Hoede C, Arnoux S, Moisset M, Chaumier T, Inizan O, Jamilloux V, et al. PASTEC: An Automatic Transposable Element Classification Tool. PLoS One. 2014;9:e91929.

4. Shapiro JA. Retrotransposons and regulatory suites. Bioessays. 2005;27:122-5.

5. Lander ES, Linton LM, Birren B, Nusbaum C, Zody MC, Baldwin J, et al. Initial sequencing and analysis of the human genome. Nature. 2001;409:860-921.

6. Smit AF. The origin of interspersed repeats in the human genome. Curr Opin Genet Dev. 1996;6:743-8.

7. Adelson DL, Raison JM, Edgar RC. Characterization and distribution of retrotransposons and simple sequence repeats in the bovine genome. Proc Natl Acad Sci U S A. 2009;106:12855-60.

8. Bejerano G, Lowe CB, Ahituv N, King B, Siepel A, Salama SR, et al. A distal enhancer and an ultraconserved exon are derived from a novel retroposon. Nature. 2006;441:87-90.

9. Feschotte C. Transposable elements and the evolution of regulatory networks. Nat Rev Genet. 2008;9:397-405.

10. Ichiyanagi K. Epigenetic regulation of transcription and possible functions of mammalian short interspersed elements, SINEs. Genes Genet Syst. 2013:88:19-29.

11. Damiani G, Florio S, Panelli S, Capelli E, Cuccia M. The Bov-A2 retroelement played a crucial role in the evolution of ruminants. Riv Biol. 2008;101:375-404.

12. Hadjiargyrou M, Delihas $N$. The Intertwining of Transposable Elements and Non-Coding RNAs. Int J Mol Sci. 2013;14:13307-28.

13. Cowley M, Oakey RJ. Transposable elements re-wire and fine-tune the transcriptome. PLoS Genet. 2013;9:e1003234.
14. Lenstra JA, van Boxtel JA, Zwaagstra KA, Schwerin M. Short interspersed nuclear element (SINE) sequences of the Bovidae. Anim Genet. 1993;24:33-9.

15. Takahashi I, Nobukuni T, Ohmori H, Kobayashi M, Tanaka S, Ohshima K, et al. Existence of a bovine LINE repetitive insert that appears in the CDNA of bovine protein BCNT in ruminant, but not in human, genomes. Gene. 1998:211:387-94.

16. Onami J, Nikaido M, Mannen H, Okada N. Genomic expansion of the Bov-A2 retroposon relating to phylogeny and breed management. Mamm Genome. 2007;18:187-96.

17. Kaukinen J, Varvio SL. Artiodactyl retroposons: association with microsatellites and use in SINEmorph detection by PCR. Nucleic Acids Res. 1992;20:2955-8.

18. Nijman IJ, van Tessel P, Lenstra JA. SINE retrotransposition during the evolution of the Pecoran ruminants. J Mol Evol. 2002;54:9-16.

19. Nilsson MA, Klassert D, Bertelsen MF, Hallstrom BM, Janke A. Activity of ancient RTE retroposons during the evolution of cows, spiral-horned antelopes, and Nilgais (Bovinae). Mol Biol Evol. 2012;29:2885-8.

20. Bibi F. A multi-calibrated mitochondrial phylogeny of extant Bovidae (Artiodactyla, Ruminantia) and the importance of the fossil record to systematics. BMC Evol Biol. 2013;13:166.

21. Arif IA, Bakir MA, Khan HA. Inferring the phylogeny of bovidae using mitochondrial DNA sequences: resolving power of individual genes relative to complete genomes. Evol Bioinform Online. 2012;8:139-50.

22. Matthee CA, Davis SK. Molecular insights into the evolution of the family Bovidae: a nuclear DNA perspective. Mol Biol Evol. 2001;18:1220-30.

23. Hassanin A, Douzery EJ. The tribal radiation of the family Bovidae (Artiodactyla) and the evolution of the mitochondrial cytochrome $b$ gene. Mol Phylogenet Evol. 1999;13:227-43.

24. Hassanin A, Douzery EJ. Molecular and morphological phylogenies of ruminantia and the alternative position of the moschidae. Syst Biol. 2003:52:206-28.

25. Loftus RT, MacHugh DE, Bradley DG, Sharp PM, Cunningham P. Evidence for two independent domestications of cattle. Proc Natl Acad Sci U S A. 1994;91:2757-61.

26. Clutton-Brock J. A Natural History of Domesticated Mammals. Cambridge, UK: Cambridge University Press; 1999.

27. Zeder MA. Domestication and early agriculture in the Mediterranean Basin: Origins, diffusion, and impact. Proc Natl Acad Sci U S A. 2008;105:11597-604.

28. Zeder MA, Hesse B. The initial domestication of goats (Capra hircus) in the Zagros mountains 10,000 years ago. Science. 2000;287:2254-7.

29. Taberlet $P$, Coissac E, Pansu J, Pompanon F. Conservation genetics of cattle, sheep, and goats. C R Biol. 2011;334:247-54.

30. Zeder MA, Emshwiller E, Smith BD, Bradley DG. Documenting domestication: the intersection of genetics and archaeology. Trends Genet. 2006;22:139-55.

31. Vigne JD, Helmer D, Peters J. New archaeozoological approaches to trace the first steps of animal domestication: general presentation, reflections and proposals. In: Vigne JD, Helmer D, Peters J, editors. Proceeding of the 9th Conference of the International Council of Archaeozoology: August 2002; Durham, UK. Oxford, UK: Oxford Books; 2005. p. 1-16.

32. Zimin AV, Delcher AL, Florea L, Kelley DR, Schatz MC, Puiu D, et al. A wholegenome assembly of the domestic cow, Bos taurus. Genome Biol. 2009;10:R42.

33. Elsik CG, Tellam RL, Worley KC, Gibbs RA, Muzny DM, Weinstock GM, et al. The genome sequence of taurine cattle: a window to ruminant biology and evolution. Science. 2009;324:522-8.

34. Reese JT, Childers CP, Sundaram JP, Dickens CM, Childs KL, Vile DC, et al. Bovine Genome Database: supporting community annotation and analysis of the Bos taurus genome. BMC Genomics. 2010;11:645.

35. Hancks DC, Goodier JL, Mandal PK, Cheung LE, Kazazian Jr HH. Retrotransposition of marked SVA elements by human L1s in cultured cells. Hum Mol Genet. 2011;20:3386-400.

36. Fukao T, Aoyama Y, Murase K, Hori T, Harijan RK, Wierenga RK, et al. Development of MLPA for human ACAT1 gene and identification of a heterozygous Alu-mediated deletion of exons 3 and 4 in a patient with mitochondrial acetoacetyl-CoA thiolase (T2) deficiency. Mol Genet Metab. 2013;110:184-7.

37. SantaLucia Jr J. A unified view of polymer, dumbbell, and oligonucleotide DNA nearest-neighbor thermodynamics. Proc Natl Acad Sci U S A. 1998;95:1460-5.

38. Zuker M. Mfold web server for nucleic acid folding and hybridization prediction. Nucleic Acids Res. 2003;31:3406-15. 
39. Cartharius K, Frech K, Grote K, Klocke B, Haltmeier M, Klingenhoff A, et al. MatInspector and beyond: promoter analysis based on transcription factor binding sites. Bioinformatics. 2005;21:2933-42.

40. Churakov G, Kriegs JO, Baertsch R, Zemann A, Brosius J, Schmitz J. Mosaic retroposon insertion patterns in placental mammals. Genome Res. 2009;19:868-75.

41. Medstrand $P$, van de Lagemaat LN, Mager DL. Retroelement distributions in the human genome: variations associated with age and proximity to genes. Genome Res. 2002;12:1483-95.

42. Wright SI, Agrawal N, Bureau TE. Effects of recombination rate and gene density on transposable element distributions in Arabidopsis thaliana. Genome Res. 2003;13:1897-903.

43. Bibi F, Bukhsianidze M, Gentry AW, Geraads D, Kostopoulos DS, Vrba ES. The fossil record and evolution of bovidae: state of the field. Palaeontol Electron. 2009;12:10A

44. Ge R-L, Cai Q, Shen Y-Y, San A, Ma L, Zhang Y, et al. Draft genome sequence of the Tibetan antelope. Nat Commun. 2013;4:1858.

45. Lei R, Jian Z, Hu Z, Yang W. Phylogenetic relationships of Chinese antelopes (sub family Antilopinae) based on mitochondrial Ribosomal RNA gene sequences. J Zool. 2003;261:227-37.

46. Kato T, Inagaki H, Kogo H, Ohye T, Yamada K, Emanuel BS, et al. Two different forms of palindrome resolution in the human genome: deletion or translocation. Hum Mol Genet. 2008;17:1184-91.

47. Kondrychyn I, Garcia-Lecea M, Emelyanov A, Parinov S, Korzh V. Genomewide analysis of Tol2 transposon reintegration in zebrafish. BMC Genomics. 2009;10:418.

48. Jobse C, Buntjer JB, Haagsma N, Breukelman HJ, Beintema JJ, Lenstra JA. Evolution and recombination of bovine DNA repeats. J Mol Evol. 1995;41:277-83.

49. Meyer TJ, Srikanta D, Conlin EM, Batzer MA. Heads or tails: L1 insertionassociated 5' homopolymeric sequences. Mob DNA. 2010;1:7.

50. Chapman RS, Lourenco PC, Tonner E, Flint DJ, Selbert S, Takeda K, et al. Suppression of epithelial apoptosis and delayed mammary gland involution in mice with a conditional knockout of Stat3. Genes Dev. 1999;13:2604-16.

51. Chapman RS, Lourenco P, Tonner E, Flint D, Selbert S, Takeda K, et al. The role of Stat3 in apoptosis and mammary gland involution. Conditional deletion of Stat3. Adv Exp Med Biol. 2000;480:129-38.

52. Humphreys RC, Bierie B, Zhao L, Raz R, Levy D, Hennighausen L. Deletion of Stat3 blocks mammary gland involution and extends functional competence of the secretory epithelium in the absence of lactogenic stimuli. Endocrinology. 2002;143:3641-50.

53. Watson CJ, Kreuzaler PA. Remodeling mechanisms of the mammary gland during involution. Int J Dev Biol. 2011;55:757-62.

54. Scribner KC, Wellberg EA, Metz RP, Porter WW. Singleminded-2 s (Sim2s) promotes delayed involution of the mouse mammary gland through suppression of Stat3 and NFkappaB. Mol Endocrinol. 2011;25:635-44.

55. Jerry DJ, Pinkas J, Kuperwasser C, Dickinson ES, Naber SP. Regulation of p53 and its targets during involution of the mammary gland. J Mammary Gland Biol Neoplasia. 1999:4:177-81.

56. Jerry DJ, Kuperwasser C, Downing SR, Pinkas J, He C, Dickinson E, et al. Delayed involution of the mammary epithelium in BALB/c-p53null mice. Oncogene. 1998;17:2305-12.

57. Jerry DJ, Dickinson ES, Roberts AL, Said TK. Regulation of Apoptosis During Mammary Involution by the p53 Tumor Suppressor Gene. J Dairy Sci. 2002:85:1103-10.

58. Matthews JR, Clarke AR. p53 mediates a default programme of mammary gland involution in the absence of STAT3. Oncogene. 2005;24:3083-90.

59. Connelly L, Barham W, Pigg R, Saint-Jean L, Sherrill T, Cheng D-S, et al. Activation of nuclear factor kappa B in mammary epithelium promotes milk loss during mammary development and infection. J Cell Physiol. 2010;222:73-81.

60. Capuco A, Akers RM. Mammary Involution in Dairy Animals. J Mammary Gland Biol Neoplasia. 1999:4:137-44.

61. Stefanon B, Colitti M, Gabai G, Knight CH, Wilde CJ. Mammary apoptosis and lactation persistency in dairy animals. J Dairy Res. 2002;69:37-52.

62. Holst BD, Hurley WL, Nelson DR. Involution of the bovine mammary gland: histological and ultrastructural changes. J Dairy Sci. 1987;70:935-44.

63. Brennan AJ, Sharp JA, Lefevre CM, Nicholas KR. Uncoupling the mechanisms that facilitate cell survival in hormone-deprived bovine mammary explants. J Mol Endocrinol. 2008:41:103-16.

64. Capuco AV, Ellis SE, Hale SA, Long E, Erdman RA, Zhao X, et al. Lactation persistency: insights from mammary cell proliferation studies. J Anim Sci. 2003;81 Suppl 3:18-31.

\section{Submit your next manuscript to BioMed Central and take full advantage of:}

- Convenient online submission

- Thorough peer review

- No space constraints or color figure charges

- Immediate publication on acceptance

- Inclusion in PubMed, CAS, Scopus and Google Scholar

- Research which is freely available for redistribution

Submit your manuscript at www.biomedcentral.com/submit 